epidemic diarrhœa two groups of organisms producing diarrhoea in puppies, the one group apparently new, and corresponding exactly with our original strain Bacillus $F$, and the other identical with Bacillus suipestifer. 2 . These groups are distinguisbable from each other, from Paratyphoid $B$, and from Bacillus suipestifer by absorption tests alone. : 3. They were not present in the stools of 100 normal children.

\section{THE TECHNIQUE OF A SIMPLIFIED FORM OF THE WASSERMANN REACTION.}

By W. D'ESTE EMERY, M.D., B.Sc. LoNo., PATHOLOGIST TO KING'S COLLEGE HOSPITAL; PATHOLOGIST To IHE CHILDREN's HOSPITAL, PADdINGTON-GREEN.

THE results of the use of the Wassermann reaction are so very satisfactory, and so many modifications of the original technique have been introduced, that the publication of a new method can only be justified on the grounds that it is simpler and quicker in application and gives as good results as any of the other processes now in use. The method that I am about to describe fulfils, I believe, these conditions. It is simple, easy, and quick in application, and, so far as $I$ have been able to test the results clinically, is most accurate. I admit fully that before a final estimate of its value can be formed it ought to be compared with the results of the original Wassermann reaction in a large series of cases. This I have not been able to do; but a process that has given accurate results in 95 per cent. at least of cases cannot be very greatly inferior to any other. I do not claim any originality for the idea of the process, which is a modification of that of Tsernugobow, whereas the details of the technique are very similar to those of Flemming. No complement is added, that which is present in the serum being sufficient. The corpuscles used are human, a point of great advantage to workers who have not access to living animals or to the slaughter-house. The only substance used which necessitates the use of animals is the immune body which joins up human complement and human red corpuscles. This is prepared by injecting thoroughly washed human corpuscles into a rabbit. It is not necessary to give more than two or three injections, which should not be at a greater interval than a week, each dose consisting of about 10 cubic centimetres of 50 per cent. emulsion, given intraperitoneally. The serum will probably not be very powerful, but this is not of much importance, since a very small amount is used in the reaction, and the serum from one rabbit will serve for many hundreds of tests. It is prepared for use as follows: The animal is bled to death and the blood collected under aseptic conditions. The serum is then standardised with human corpuscles and fresh human serum so as to determine the minimal hæmolytic dose under the conditions of the experiment. It is then diluted with sterile normal saline solution in the proportion indicated in the standardisation experiments, pipetted off into sterile vaccine bulbs $\left(\frac{1}{2}-1\right.$ cubic centimetres in each), sealed, and heated to $60^{\circ} \mathrm{C}$. for half an hour. If there is any doubt as to its sterility the heating should be repeated on two other successive days.

The method of standardisation will be seen from an example. The requisites are: 1. A 20 per cent. emulsion of human red corpuscles in normal saline; it should have been rewashed at least three times. 2. Fresh normal human serum. 3. Normal saline. 4. The serum to be tested, which must have been previously heated to $60^{\circ} \mathrm{C}$. for half an hour. The following tubes were prepared :-

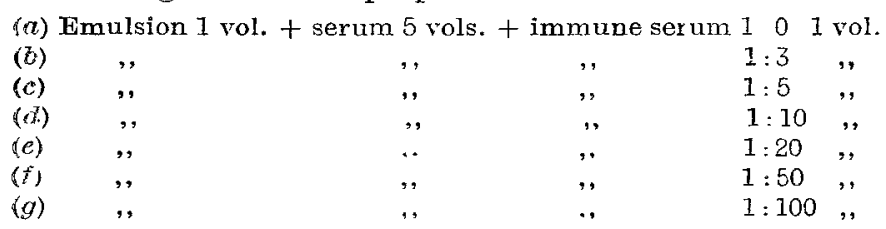

The mixtures of the ingredients were made by means of an ordinary Wright's opsonic pipette with a unit mark about one inch from the end and the serum dilutions prepared in a similar way. The mixtures were placed in a small test-tube about $\frac{1}{8}$ th inch internal diameter, well-stirred with the pipette, and incubated at $37^{\circ} \mathrm{C}$. for one hour. (They were thoroughly stirred after half an hour.) The following was the result: with $a, b, c$, and $d$ there was complete hæmolysis; with $e$ there was a trace of hæmolysis and much agglutination; with $f$ there was agglutination, but no hæmolysis ; and with $g$ there was partial a grglutination. I nis serum was only diluted 1 in 4 for use, so as to make sure of there being an excess of immune body in the conditions of the experiments. I may say that the 5 volumes of serum contain a large excess of complement.

The blood for the test is collected in the ordinary way from a skin puncture, and about $\frac{1}{4}$ cubic centimetre of blood is ample, even if the test has to be repeated. I may point out, what $I$ find is not known as generally as it should be, that if a large crop of serum is desired it is advantageous (a) that the blood shall not be allowed to cool after it is collected, and $(b)$ that the clot shall be separated from the sides of the vessel in which it is contained, so as to allow of free retraction. To meet the former indication it is a good plan to put the pipette in the incubator as soon as possible after it has been filled; to meet the latter shake the clot as soon as it has formed towards the curved end of the pipette and back again.

The "antigen" used has been the ordinary alcoholic extract of normal heart, prepared by grinding up a weighed amount of heart muscle with four time the number of cubic centimetres of absolute alcohol that there are grammes of muscle, allowing it to stand 24 hours and repeating the grinding, repeating the maceration for another 24 hours, after which it is heated to $60^{\circ} \mathrm{C}$. for one hour. It must be quite clear when used, a little being withdrawn from the top layer by means of a pipette. It is diluted with nine times its volume or more, as determined by experiments, of normal saline for use. Before use and afterwards occasionally it is in the highest degree necessary to test this extract. To be of use it should (a) give complete deviation of the complement in a known case of late secondary or early tertiary syphilis, even when 1 volume of fresh normal serum from a healthy person is added; and (b) used in the conditions of the test about to be described should cause very little absorption (or destruction?) of com. plement in a normal blood. This is tested simply as follows: Two tubes are prepared, of which the first contains 1 volume of normal serum, 4 volumes of normal saline, and the second 1 volume of the same serum, and 4 volumes of the diluted extract. These are incubated together for half an hour, and to each is added 1 volume of the immune serum prepared as above, and an excess ( 5 volumes is usually enough) of 20 per cent. emulsion of washed human corpuscles. The incubation is repeated for one hour, the tubes being stirred once or twice. At the end of that time they are centrifugalised, and a definite quantity of the clear blood-stained fluid pipetted off and examined in a hæmoglobinometer. In a good extract there should be no difference between the two, and this is sometimes the case. Lsually there is a slight difference which naturally tends to interfere with the accuracy of the reaction. This, of course, is common to all methods. I am not prepared to give exact figures, but if there were a marked difference between the amount of hamoglobin dissolved in the two tubes the extract should be discarded or tested again at a higher dilution.

The only other ingredients-normal saline and a 20 per cent. emulsion of human corpuscles, well rewashed-do not call for mention.

The only apparatus required consists of (1) a Wright's pipette, rather wide-i.e., about 1 millimetre in internal

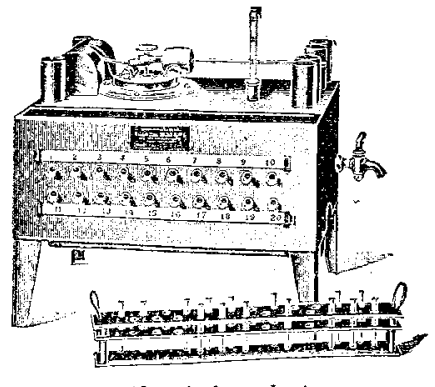

Author's incubator.

diameter, with a 1 unit mark about 1 inch from the end and a 4 unit mark; (2) a series of small test-tubes about $\frac{1}{8}$ th inch internal diameter and 2 inches long; and (3) an incabator. Grood results can be obtained with an ordinary biological incubator at $37^{\circ} \mathrm{C}$,, and, indeed, most of my results have been obtained in this way. It is. however, a very great advantage, as will appear subsequently, that the mixtures shall not be cold at the 
final stage. To avoid this the stand in which the tubes are held may be placed in a water bath at $37^{\circ} \mathrm{C}$., and the last addition made whilst they remain in situ. I have recently devised a form of incubator specially for the purpose. It is a modification of Hearson's opsonic incubator, part of the top of which is removable and forms a stand adapted to 24 tubes such as $I$ have described. This can be removed and placed on a table and the tubes filled in the ordinary way. It can then be returned to the incubator for the rest of the process. The final additions-of immune serum and emulsion-can be made with the tubes in sitk and surrounded by warm water. The emulsion and serum may be warmed in the incubator, so that the test-mixture need never be cooled at all. I think bacteriologists will find this incubator useful for many purposes.

The process is as follows. Prepare a Wright's pipette with a 1 and 4 unit mark. Place in each of the tubes of the front row of the incubator 4 units of normal saline solution; these are to serve as controls in order to make sure that the serum to be tested contains sufficient free complement and that the hæmolytic system is in working order. In each of the tubes of the back row 4 units of the diluted extract are placed and the pipette carefully washed out.

One unit of the first specimen of serum is now added to each of the first two pairs of tubes, that containing the normal saline solution receiving its addition first, then that containing the extract; this is to avoid carrying over a little extract into the control. In each case the fluids are thoroughly mixed by repeatedly sucking them into the pipette and expelling them, and in each case it is advisable to see that the mixture forms a continuous column. The pipette is then rinsed out with normal saline solution, and the process repeated with as many sera as are to be tested.

Then the hæmolytic system is prepared thus. Take 1 unit of red corpuscles which have been centrifugalised three or four times, and mix them with four times their volume of the prepared rabbits' serum. This will be more than enough to saturate them with amboceptor and also with agglutinin. Put them in the incubator so as to hasten the combination of the corpuscles and the antibodies.

The final stage of the test consists in the addition of these sensitised and agglutinated corpuscles to the tubes containing the diluted serum. A point which I particularly wish to emphasise is that it is not necessary to wait for an hour before this addition is made. Numerous tests have shown me that the combination in the extract, tube is complete in five minutes after it has reached $38^{\circ} \mathrm{O}$., and almost complete in two and a half minutes. Of course, if the tubes are incubated in air these times are greater, since the tubes and their contents take an appreciable length of time to get heated up to this temperature; but when the two substances are placed in a narrow tube surrounded with warm water the combination takes place, as I have stated, in five minutes. This is one of the advantages of the incubator made by Messrs. Hearson for me. When a series of half-a-dozen sera have to be tested the first is ready for the sensitised corpuscles as soon as the last mixture has been prepared.

The sensitised corpuscles are strongly agglutinated and form a mass at the bottom of the tube. They are thoroughly stirred up by alternately sucking them into the pipette and expelling them into the tube (still surrounded by warm water in the incubator), and then one unit of the mixture is added rapidly (so that agglutination does not have time to take place again) to each tube of diluted serum. In making these additions, I am in the habit of making the first of each pair to the tube containing serum and extract, then to that containing serum and saline; by so doing I avoid carrying over a trace of complement to the second tube. In each case the corpuscles are very thoroughly mixed in.

The great practical advantage of the method is now apparent. The hæmolysis of the first tube-that containing no extract -is almost instantaneous; warm fluid containing complement has added to it warm corpuscles fully sensitised. On the other hand, the clearing of the second tube (in the case of a positive reaction) is very rapid; the corpuscles are saturated with agglutinin, and being kept warm clump with great rapidity. If the clumping takes place too quickly it may be advisable to give it a second stir before taking the final reading, though, as a matter of fact, I have rarely seen this convert a negative into a positive test.

The great advantages that I wish to claim for this method are its simplicity and rapidity. The latter is most striking.
Given that the preparations have been made, the corpuscles washed, and the extract diluted, a serum can be tested within 10 or 15 minutes. As compared with Flemming's method (the modification most used in this country), it has the additional advantage of being (as far as $I$ have seen from an examination of over 200 sera) universally applicable. I have not yet encountered a specimen of serum which did not give abundant hæmolysis when tested in the manner described without the addition of extract within 48 hours of its being collected. ${ }^{1}$ As far as my experience goes, this is frequently the case with Flemming's method, more frequently than some experimenters find. Thus in seven specimens of serum three gave no trace of hæmolysis with sheep's corpuscles, but all gave a perfect reaction when tested by my method. Where Flemming's method is applicable it seems to give identical results, though less obvious, the amount of hæmolysis being less. I am aware that Flemming's method can be made applicable in all cases by the addition of known hæmolytic serum, but this introduces a complication which at the present time has been unnecessary with my own. Another convenience is, of course, the fact that normal human corpuscles are employed.

An advantage over the classical Wassermann reaction is the fact that the serum is not heated. This is not only a convenient simplification of the method, but is of theoretical advantage.

The only objection to the method is the variation in the amount of complement present in the serum of different persons and of the same person from hour to hour. This is most marked, and, of course, it prevents any quantitative work from being done by the method except by a somewhat laborious process involving the use of a considerable amount of serum. As against this we must set the convenience and rapidity of the test, regarded as a qualitative rather than as a quantitative one, and the good results that it has given in actual practice.

It has now been used in over 200 cases, and the results have been uniformly excellent. In my first 100 cases, in which the diagnosis was known or supposed to be known accurately to the medical man under whose care the patient was, 40 were patients in whom there was no suspicion of syphilis, and all were negative. They included cases of abscesses, boils, abscess of the lung, phthisis, surgical tubercle, malignant disease (both carcinoma and sarcoma), gonorrhoa (both acute and chronic), soft sore, lupus, pernicious anæmia, diabetes, jaundice, typhoid fever, \&c. Of the 60 known cases of syphilis, five were primary; three gave the reaction, one of them in a man who averred that the chancre had only appeared four days previously. This, of course, is much earlier than the reaction usually occurs, and I think there must have been an error on the part of the patient. Ten only were examined in the secondary stage ; all without exception were positive. Thirteen were congenital cases and all were positive ; in three of these cases the apparently healthy mother was tested and found to give the reaction. Two cases of tabes and four of general paralysis were examined; all positive (this refers to the blood, not to the cerebro-spinal fiuid, in which I have had but little experience). The remaining 23 patients had various forms of tertiary lesions (mostly gummata, ulcers of the skin, and joint lesions) or were old cases with a perfectly clear history of syphilis, but without active disease at the time of the examination. Twenty-one (all except two) gave positive reactions. Of the two remaining, one was a patient with a history of syphilis 36 years ago, but with no active symptoms for many years. The last patient was the only one in which a real failure had to be recorded. The physician in charge of the case arrived at the diagnosis by a process of exclusion, but was quite convinced of its accuracy. ${ }^{2}$ The patient showed no signs of improvement after a month's antisyphilitic treatment, and denied all history of the disease in a most emphatic manner, and gave no reaction. Thus of 60 cases of syphilis, two early primary cases, one case of 36 years' duration, and one which must be called at the least doubtful, gave no reaction. I may say that in most of the cases recorded I did not know the diagnosis until the examination

1 Since writing this I have found one specimen (from a case of spleno-metullary leueocythemia), which crused but rery slight hamolysis on the only occasion on which it was tested.

2 He has since come to the conclusion, on clinical grounds alnne, that he case is not syphilitic in nature. The serum diagnosis is therefore probably correct. 
was made. These results are, I think, sufficiently striking to prove the practical value of the method.

PS.-Since writing the above an experience of more than another 100 cases has convinced me that the method yields results which are decidedly better than those claimed for the standard Wassermann test. Regarding every case as a failure in which the result does not accord with the clinical diagnosis, I still obtain more than 95 per cent. of successes, as compared with the 85 per cent. usually quoted. I have examined 12 casss of tabes; all reacted, showing 100 per cent. of correct results in place of the 50 per cent. in which the reaction is supposed to be present when tested by Wassermann's method.

\section{$\triangle$ CASE OF CEREBELLAR H屟MORRHAGE}

BY A. E. IVILSON HIRD, M.R.C.S. ENG., L.R.C.P. LOND.,

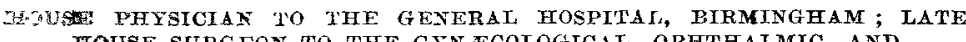
POUSE SURGEON TO THE GYN_XCOLOGICAL, OPHTHALMYC, AND AURAL DEPARTMENTS OF THF, GENERAL HOSPITAL, BIRMINGFAM.

Tur patient, a boy, aged 10 years, was admitted to the General Hospital, Birmingham, on June 3rd, 1910. The following history was obtained from the schoolmaster and the patient's father. The boy was first taken ill on May 28th, 1910. He complained of severe colicky pains in the abdomen and was sent to bed. Next day he was worse and somplained of severe frontal headache; he also vomited several times. Two days later he screamed at intervals of about half an hour in addition to his other symptoms. He was quite conscious. Daring 24 hours previous to admission 需e commenced to get "drowsy," although on the morning he was removed to hospital he was able to answer quesficns. The child belonged to a large family of healthy children. The mother was very neurotic; the father was quite healthy. Six years ago the patient was knocked down by a motor-car and one wheel of the car passed over his head, and he was said to have sustained a fracture of the base of the skull. His mental condition had always ween poor. Ife had had no other illness. There was no history of phthisis in the family and no history of recent ajury could be obtained.

Yn admission the patient lay in bed on his right side; head retraction was well marked, the arms were folded on che chest, and the knees were drawn up so as to nearly touch the abdomen. The abdomen was markedly scaphoid. He resented any interference and cried out when touched; if moved into any fresh position he soon resumed his old ativitude. The patient was quite unable to answer any guestion pat to him. Both eyes were wide open and both perpils were widely dilated; there was no squint noticeable. Poth cheeks had a pronounced malar flush. The patient's breath was very fotid and the tongue was coated with a thick brown fur over the dorsum. His temperature was 98.5० F. The pulae was 84 per minute, of full volume, low tension, and regular.

on examination the patient had well-marked head retrac. Gicn. Fxamination of the cervical spine distressed him. Muscular power on both sides of the body was apparently equal. There was no paralysis or paresis of either the upper or the lower extremities. The body was fairly well nourished. Sensation appeared to be unaffected. On examining the eyes the papils reacted sluggishly to light; no nystagmoid movements were observable. It was impossible to make a proper examination of the optic discs as the patient resented a strong light being thrown into his eyes; from a fleeting glance the discs appeared mormal and the veins were congested. No lesion of the Branial nerves could be detected. Urine was passed involuntarily. The bowels had not been opened since 3.dmission On examination of the patient's reflexes, both plantai reflexes were flexor. There was no ankle clonus on either side. Both knee-jerks were absent. Kernig's sign

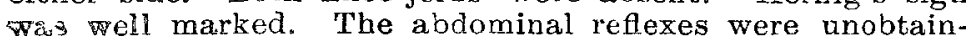
able. The supinator triceps and the jaw jerks could not be obtained. Nothing abnormal could be detected or. examination of the chest or abdomen. Examination of a catheter specimen of urine revealed no pathologioal condition. Seen the same evening at 8.30 the patient was apparently sleeping; both eyelids were semi-
closed, and he lay in the same attitude as on admission. From time to time he gave vent to loud cries. Seen again the following morning, the patient's condition was unaltered. Lumbar puncture was performed at 11.30 ; the needle was introduced between the first and second lumbar vertebræ with considerable difficulty, as the patient writhed about in the bed. The fluid was found to be under considerable pressure and squirted from the orifice of the needle. The fluid was quite clear; a little over 10 cubic centimetres were with drawn. Nothing pathological in its constitution was found on examination. At 2.30 P.M. the same day the patient suddenly stopped breathing. All efforts to restore respiration were unavailing. The pulse was present at the wrist for three-quarters of an hour after the cessation of respira. tion; it improved for a time while artificial respiration was being performed, but at the end of three-quarters of an hour the heart ceased to beat.

Necropsy.-The result of the post-mortem examination was as follows. The body was well nourished. Rigor mortis was present in the upper and lower extremities. His height was 4 feet $1 \frac{1}{2}$ inches, his weight was 3 stones $4 \frac{1}{2}$ pounds, and his chest measurement was 23 inches. With regard to the respiratory system, the epiglottis was normal, the trachea and bronchi were injected and contained frothy mucus, and the pleuræ was normal except for some slight adhesions to the diaphragm on the right side. As to the lungs, the weight of the right was 7 ounces and of the left 9 ounces; they showed marked emphysema, which was most noticeable just below the pleuræ, and they were congested and slightly cedematous. There were no signs of pneumonia. The lymphatic glands at the roots of the lungs were not enlarged. With regard to the circulatory system, the capacity of the pericardium was 177 cubic centimetres. The heart weighed $4 \frac{3}{4}$ ounces. The pericardium contained a small amount of free fluid; there was no pericarditis. The mitral and tricuspid valves of the heart were normal. Each of the pulmonary and aortic valves had only two cusps, one large one, and one about the normal size. The appearance was as if the two anterior pulmonary cusps had fused to form one large one, and as if the two posterior aortic cusps had done likewise. Both coronary arteries were normal, so also were the large bloodvessels. The cavities were not enlarged. The heart muscle was normal in colour and in consistence. The cesophagus was normal. The stomach and small intestines were all healthy. Small post-mortem intussusception was found in the jejunum. The mucous membrane of the large intestine was injected and showed well-marked post-mortem staining. The liver weighed 28 ounces; it was very congested, dark purple in colour, very firm, and not easily friable. The gall-bladder and bile-ducts were normal. The pancreas was normal. The kidneys were both very firm, the left being the larger; the right weighed $2 \frac{1}{2}$ ounces and the left 3 ounces; the capsules stripped readily. $\quad F_{0 \text { tal lobulation was well marked. There }}$ was marked congestion of both organs. The cortex and medulla were normal in thickness. There was no nephritis. The ureters and bladder were normal. As to the ductless glands, the thyroid was normal. The spleen weighed $3 \frac{1}{2}$ ounces, was firm, and dark red in colour; the capsule was thick and tough. The suprarenals were normal. Microscopical sections of the various organs revealed no pathological change. With regard to the central nervous system, the brain weighed 43 ounces. The cerebral membranes did not show any meningitis. The brain showed some cedema of the cerebral hemispheres and internal hydrocephalus. Behind and external to the lateral ventricle, in the left side of the cerebrum, was a small cystic area of about the size of a pea showing the remains of an old hremorrhage. In the right lateral lobe of the cerebellum there was a large hæmorrhage of about the size of a golf ball, the blood clot being black and shiny with two whitish firmer areas. The substance of the cerebellum round the clot was darker than normal. The hæmorrhage had almost reached the surface posteriorly. The blood-vessels of the brain were quite healthy. Microscopical examination did not show any pathological condition. No trace could be found of the injury received to the cranial bones by his accident six years ago.

The above case was of peculiar interest from the fact that it showed many of the typical signs of a basal meningitis which indeed was the provisional diagnosis. I am indebted to Sir Robert M. Simon for permision to publish the case.

Birmingham. 